\title{
Sistematização das atividades em comunidades criativas. Estudo de caso: Núcleo de Abordagem Sistêmica do Design (NASDesign) no Brasil
}

\section{SYSTEMATIZATION OF ACTIVITIES IN CREATIVE COMMUNITIES. CASE : NÚCLEO DE ABORDAGEM SISTÊMICA DO DESIGN (NASDESIGN) IN BRAZIL}

Juliane Marcal da Silva Prodanov ${ }^{1}$ Luiz Fernando Figueiredo ${ }^{2}$ 


\section{Resumo}

O objetivo deste artigo é demonstrar o processo de sistematizar as atividades desenvolvidas pelo Núcleo de Abordagem Sistêmica do Design (NASDesign)- Brasil da Universidade Federal de Santa Catarina (UFSC). O NASDesign desenvolve trabaIhos com grupos produtivos, cooperativas e associações de Santa Catarina por meio de projetos sociais. O Centro é responsável pelo desenvolvimento de projetos envolvendo ferramentas, metodologias e estratégias de design para desenvolver Visual Sistema de Identidade (VIS), manuais de identidade visual, embalagens, rótulos, os trabalhos que envolvem a ergonomia, desenvolvimento de produtos, entre outros.

Palavras-Chave: : NASDesign. Grupos Produtivos Locais. Design. Sistematização das atividades.

ISSN: 1808.3129

\section{Abstract}

The purpose of this article is to demonstrate the process of systematizing the activities developed by Núcleo de Abordagem Sistêmica do Design (NASDesign) of the Universidade Federal de Santa Catarina (UFSC). The NASDesign develops works with productive groups, cooperatives and associations of Santa Catarina through social projects. The Center is responsible for developing projects involving tools, methodologies and design strategies to develop Visual IdentitySystem (VIS), Visual Identities manuals, packaging, labels, jobs involving ergonomics, product development, among others.The aim was to strengthen the NASDesign the identity of these productive groups, associations and cooperatives of Santa Catarina, through the Enabling Design Process for Local Initiatives in order to improve the positioning of their products on the market and enhance the roots of their local traditions. With this process, it was possible to build experiences with communities in order to interpret subjective characteristics that represented the experiences of these families in their values and aesthetics. The result was the conformation of visual identity for all, as a coat of arms of family farming, which brought social motivation and sense of belonging, in addition to greater acceptance to design practices. With this opening, is much easier to insert other types of social programs such as the inclusion of new services, interfaces and tools that better organize production to a sustainable reality.

Keywords: NASDesign, Groups Local Production, Design, Systematization of activities, creative communities. 


\section{INTRODUÇÃO}

O objetivo do trabalho é relatar o método utilizado no Núcleo de Abordagem Sistêmica de Design - NASDesign e como seu método empregado resulta em resultados positivos para as comunidades criativas auxiliadas.

Para compreender e buscar melhorias no processo de desenvolvimento de um produto em uma organização é necessário compreender seu funcionamento. Segundo CEAAL (1992), entendemos a sistematização como um processo permanente, cumulativo, de criação de conhecimentos a partir da experiência de intervenção em uma realidade social, como um primeiro nível de teorização sobre a prática.

O Núcleo de Abordagem Sistêmica do Design tem como objetivo primário a introdução do design em empresas de cunho familiar deste modo valorizando seus produtos e expandindo seu mercado consumidor. O NASDesign busca valorizar não só o produto mas o meu onde este produto esta inserido (cidade, região, pessoas) . E com intuito de demonstrar que a proposta do núcleo de introduzir uma sistematização das atividades garante um suporte adequado aos projetos.

Segundo Manzini (2008), o projeto é capaz de promover o desenvolvimento das comunidades, através da compreensão de suas formas criativas de organização / produção, o que permite a criação de conhecimento, técnicas e ferramentas para ser aplicado em outras organizações.

No contexto das comunidades criativas, o projeto pode proporcionar significativas inovações em modelos de produção, transformando o cotidiano dos participantes do processo de produção, dando-lhes a oportunidade de se manter ativo e útil .

A partir da criação de marcas, identidades consideradas que representam a região, os membros dessas comunidades estão a valorizar suas próprias atividades e as realizadas no local.

\section{METODOLOGIA}

Com base nos seus objetivos, esta pesquisa pode ser classificada como pesquisa exploratória. Referente ao delineamento da pesquisa, para o entendimento dos temas abordados na fundamentação teórica, esta pesquisa pode ser classificada como pesquisa bibliográfica, pois se baseia em material já elaborado - livros de literatura corrente e de referência, artigos científicos e periódicos (GIL, 2002).

Torna-se relevante destacar que na pesquisa bibliográfica foram obtidas informações e dados a cerca das definições referentes aos grupos produtivos locais, ao design, à Gestão de Design, bem como, a abordagem sistêmica.

Referente ao interesse prático desta pesquisa pode ser caracterizada como pesquisa aplicada, ou seja, "[...] objetiva gerar conhecimentos para aplicação prática e dirigidos à solução de problemas específicos." (SILVA; MENEZES, 2005, p.20).

No que se refere à abordagem do problema, considera-se como uma pesquisa qualitativa, pois lida com a interpretação de fenômenos e com a atribuição de seus significados. 
Além disso, esta pesquisa pode ser classificada como estudo de caso, pois consiste no estudo de um objeto, "[...] de maneira a permitir o seu conhecimento amplo e detalhado" (GIL, 2007, p. 72), visando analisar como proporcionar a partir da Gestão de Design, com base em uma abordagem sistêmica, a sistematização das atividades do NASDesign.

Em estudos de caso pode-se recorrer a variadas técnicas de coleta de informações, como observações e entrevistas, para o estabelecimento de um diagnóstico, de uma organização ou fazer sua avaliação por uma razão específica.

Sendo assim, a presente pesquisa foi elaborada para possibilitar a expansão do conhecimento das teorias destacadas neste artigo.

\section{FUNDAMENTAÇÃO TEÓRICA}

\section{NASDesign}

O NASDesign localizado no Centro de Comunicação e Expressão (CCE) da UFSC é um laboratório de pesquisa em design. Há 6 anos, o grupo trabalha com comunidades produtivas de Santa Catarina, dentre elas a COLIMAR, onde projetos desenvolvidos com o auxílio da UFSC e demais parceiros, permitem o exercício ativo do designer nas produções locais.

O NASDesign utiliza metodologias de revisão bibliográfica, pesquisa-ação (DIONNE, 2007) e abordagem sistêmica de design, com objetivo de desenvolver projetos sistêmicos que resultam no desenvolvimento de interfaces gráficas, design de serviços e criação de redes, visando à sustentabilidade de seus processos.

Além disso, o núcleo busca os produtores locais em situação emergente, para proporcionar o desenvolvimento econômico, social e ambiental.

Justamente por isso o NASDesign trabalha a partir de necessidades e problemas utilizando-se da pesquisa e do desenvolvimento para aplicar os conhecimentos em design.

A COLIMAR procurou o NASDesign para melhorar o seu desenvolvimento e o andamento da sua produção. Porém, ao se iniciar um projeto deste tipo, o núcleo objetiva por meio do design, desenvolver produtos e serviços mais adequados à realidade de mercado local e regional. Além disso, visa intervir por meio dos pensamentos de design (metodologias de design) nas atividades da cooperativa, requerer a modificação social por meio da valorização do produto e serviço, identificar e integrar os grupos produtivos locais no processo para o desenvolvimento local, buscar a gestão de sustentabilidade e gestão sistêmica que mais se adéque à realidade local e desenvolver uma plataforma habilitante de integração social para a gestão da informação.10 Congresso Brasileiro de Pesquisa e Desenvolvimento em Design, São Luís (MA)

Neste contexto, o designer pesquisador deve também desenvolver seu lado de mediador do conhecimento, já que pretende incentivar novos tipos de hábitos e novas aprendizagens.

O intuito é fazer não só com que os produtos da COLIMAR ganhem competiti- 
vidade de mercado, mas também que ganhe confiança em colocar seus produtos a competir junto com os demais. Este é um ponto estratégico do design, pois ao mesmo tempo em que traz motivação social às pessoas, mostrando a importância de sua atuação e fazendo-se entender os conceitos de design, também abre espaço para a incorporação de outros programas.

Isso só é possível, porque essas práticas fazem com que os envolvidos nesse processo tornem-se mais confiantes, fazendo com que tragam contribuições efetivas ao seu dia-a-dia, sem provocar mudanças profundas em suas tradições e cultura. Esta é a abertura adequada, que permite que o global interfira no local, seja com sua tecnologia ou conhecimento científico.

Sendo assim, o objetivo do NASDesign é propor, no fim de seu processo de design, soluções sistêmicas que satisfaçam as necessidades da comunidade, favoreçam à prática de iniciativas locais e, partindo de um processo também de aprendizagem, permitam que atores sociais desenvolvam uma consciência sustentável. A intenção é favorecer o fortalecimento das práticas locais, com o perduro de suas ações às gerações futuras.

De acordo com Manzini (2008), o design é capaz de potencializar o desenvolvimento de comunidades, através do entendimento de suas formas criativas de organização/produção, o que possibilita a criação de conhecimentos, técnicas e ferramentas a serem aplicadas nas demais organizações.

No contexto de comunidades criativas, o design pode proporcionar inovações significativas nos modelos de produção, transformando o cotidiano dos participantes do processo produtivo, dando-lhes a oportunidade de se manterem ativas e úteis.

A partir da criação das marcas, consideradas identidades que representam a região, os integrantes dessas comunidades passam a valorizar suas próprias atividades e as desenvolvidas no local.

\section{Abordagem sistêmica}

A estratégia de abordagem sistêmica do design provém da prática exercida pelo Núcleo de Abordagem Sistêmica do Design (NASDesign).

Para a atuação do design em comunidades criativas, MANZINI (2008) propõe três formas de interação:

- Bottom-up: de baixo para cima, pela participação ativa das pessoas interessadas;

- Top-down: de cima para baixo, pela intervenção de instituições externas;

- Peer-to-peer: entre pares, pela troca de informações entre organizações similares.

A adoção de uma abordagem sistêmica do design serviu como ferramenta para facilitar essas três formas de interação.

A abordagem sistêmica como conceito, sob o ponto de vista de Chiavenato (1993), é a ênfase da teoria moderna sobre o dinâmico de interação que ocorre dentro da estrutura de uma organização.

Para Bistagnino (2009), a abordagem sistêmica do design permite, portanto, alargar as referências, não se limitando ao produto. A base da abordagem sistêmica é 
cercar-se da natureza e das suas dinâmicas de funcionamento.

Então, a prática projetual de design pode ser considerada mais que um sistema relacionado à estratégia e administração empresarial, pois esta também possui capacidade de influência sobre o usuário, na sua motivação pessoal e profissional, bem como, nos colaboradores do processo. Estas inter-relações entre os projetos e os profissionais de design são necessárias para o desenvolvimento de um projeto, reforçando assim, que a diversidade dos profissionais aliada à integração com outros tipos de atividades, como engenharia, artes, administração entre outros, é capaz de formar um sistema. Sendo assim, o foco seria o processo de interação entre as partes que constituem uma estrutura, não ocasionando um deslocamento da ênfase do todo.

Em um sistema (Figura 1) o ambiente funciona a partir do constante fluxo de entradas (inputs) que incluem materiais, informações, conhecimento, instalações, pessoas entre outros; dentro dos limites de projeto por meio do planejamento e controle de melhorias; e de saídas (outputs), incluindo produtos e serviços.

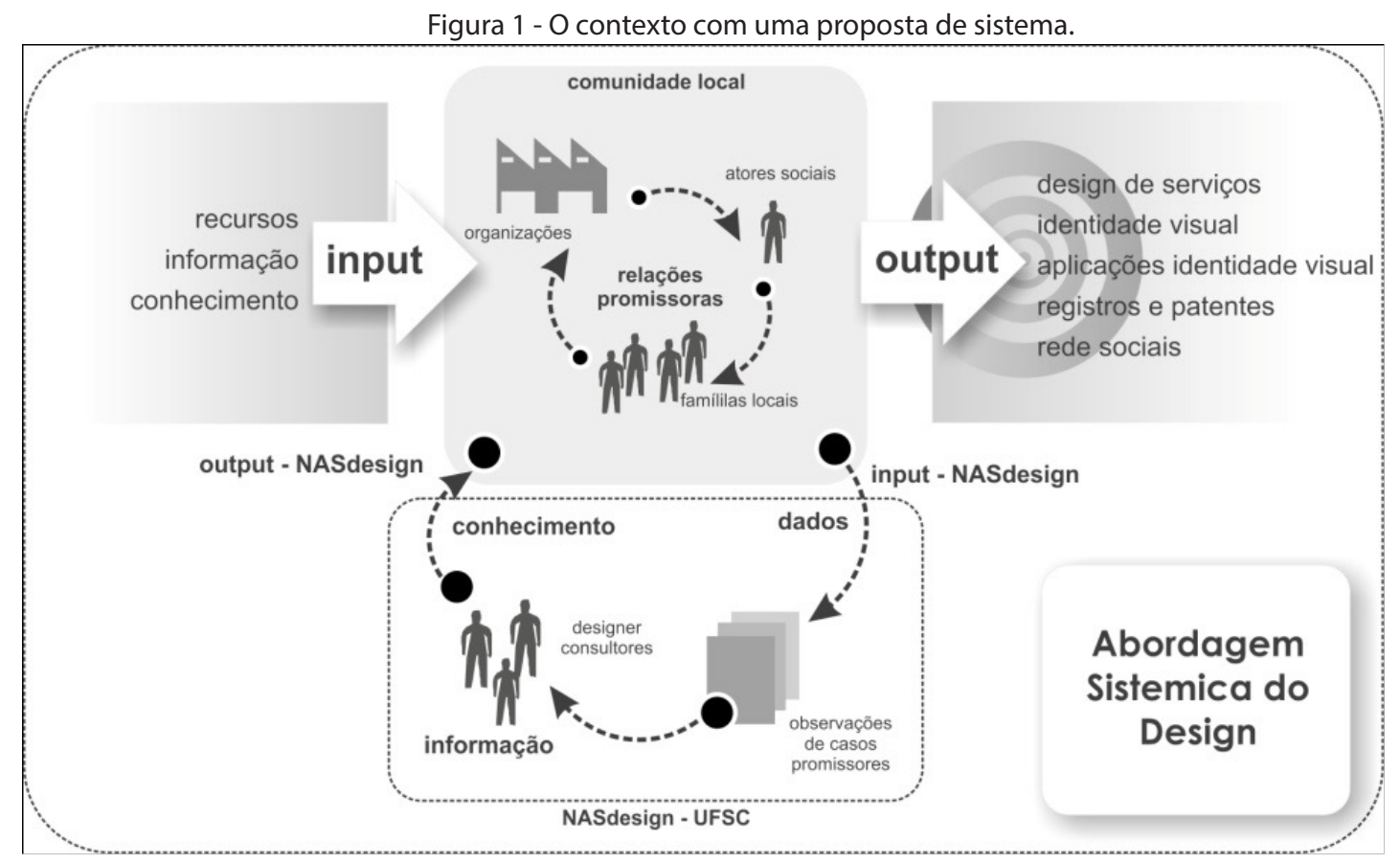

Fonte: NasDesign.

Andrade et al (2006) menciona que a adoção de abordagens sistêmicas na teoria organizacional e nas ciências relacionadas com administração vincula-se estreitamente ao crescimento da complexidade das organizações humanas, o qual trouxe a necessidade de melhorar a capacidade de administrar e solucionar problemas cada vez mais complexos. Isso fez se constituir um ambiente cada vez mais favorável à adoção de ideias sistêmicas na administração de organizações sociais e de produção.

A abordagem sistêmica utilizada pelo NASDesign se resume à integração do designer com atores sociais, que identifica ações promissoras que podem ser aplicadas em uma comunidade e verifica a capacidade dos atores em desenvolver serviços por meio da habilitação e capacitação, melhorando a produção e validando suas ações.

O NASDesign busca os produtores locais em situação emergente, para propor- 
cionar o desenvolvimento econômico, social e ambiental por meio de projetos com a Universidade e demais parceiros, e a partir de necessidades e problemas utilizando-se da pesquisa e do desenvolvimento permite o exercício ativo do designer.

Sendo assim, o NASDesign, primeiramente observa esses grupos de produtores locais que se organizam por iniciativa própria, por meio da sinalização das demandas, problemas e oportunidades, avaliando as suas necessidades por meio da identificação de causas para que sejam propostas atividades de projeto visando a solução desses problemas.

Nesta pesquisa a abordagem sistêmica contribuiu para a visualização de um panorama holístico, entendendo a como um sistema aberto, aonde as interferências ou problemas detectados podem ser relativos às interferências e as relações internas e externas dos Grupos produtivos e do sistema formado pela gestão de design.

Os atores sociais envolvidos são a maior fonte de informação para o delineamento de um panorama e para a avaliação da gestão de design extraindo indicadores para introdução de melhorias e inovações no processo de gestão de design.

\section{Sistematização das atividades do NASDesign}

A sistematização pode contribuir nas dimensões econômica, social e ambiental, pois favorece o intercâmbio de experiências, tanto para que a equipe tenha melhor compreensão de seu trabalho quanto para adquirir conhecimentos teóricos a partir da prática.

O Núcleo de Abordagem Sistêmica do Design (NASDesign), realiza investigações e discussões acerca da abordagem sistêmica do design, com o intuito de disseminar o entendimento de suas novas dimensões práticas e teóricas. Os bolsistas de graduação e pós-graduação em design que pertencem ao Núcleo pesquisam assuntos relacionados à inovação social, ao design responsável, às comunidades criativas e a outros aspectos ligados à sustentabilidade.

O NASDesign é certificado pela instituição no diretório dos grupos de pesquisa do Brasil-CNPQ e faz parte do grupo DESIS-Brasil, conectado à rede DESIS-International ${ }^{1}$.

Desde o ano de sua criação, 2006, o grupo trabalha com grupos produtivos pertencentes às comunidades do Estado de Santa Catarina, e a partir do auxílio da UFSC e demais parceiros, permitem o exercício ativo do designer. Em vista disso, o NASDesign utiliza metodologias de revisão bibliográfica, pesquisa-ação (DIONNE, 2007) e abordagem sistêmica de design, com objetivo de desenvolver projetos sistêmicos que resultam no desenvolvimento de interfaces gráficas, design de serviços e criação de redes, visando à sustentabilidade de seus processos.

O NASDesign e sua equipe de pesquisa têm seu olhar voltado para a sociedade e a partir desse contato percebem as demandas, os problemas e as oportunidades, além disso, identificam as causas das demandas não atendidas e promovem ações visando a solução de problemas por meio de atividades de projeto (Figura 2). 
Figura 2 - Olhar para a sociedade e propor ação.

Fonte: NasDesign.

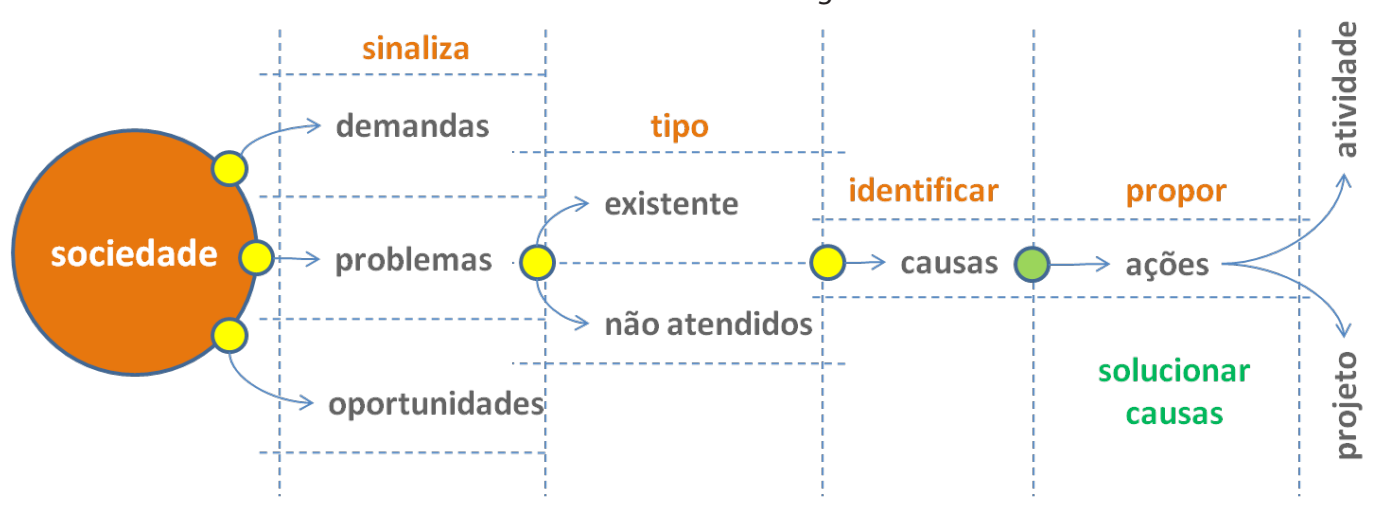

Figura 3 - Metodologia Péon com uma abordagem sistêmica.

Fonte: PÉON, 2001

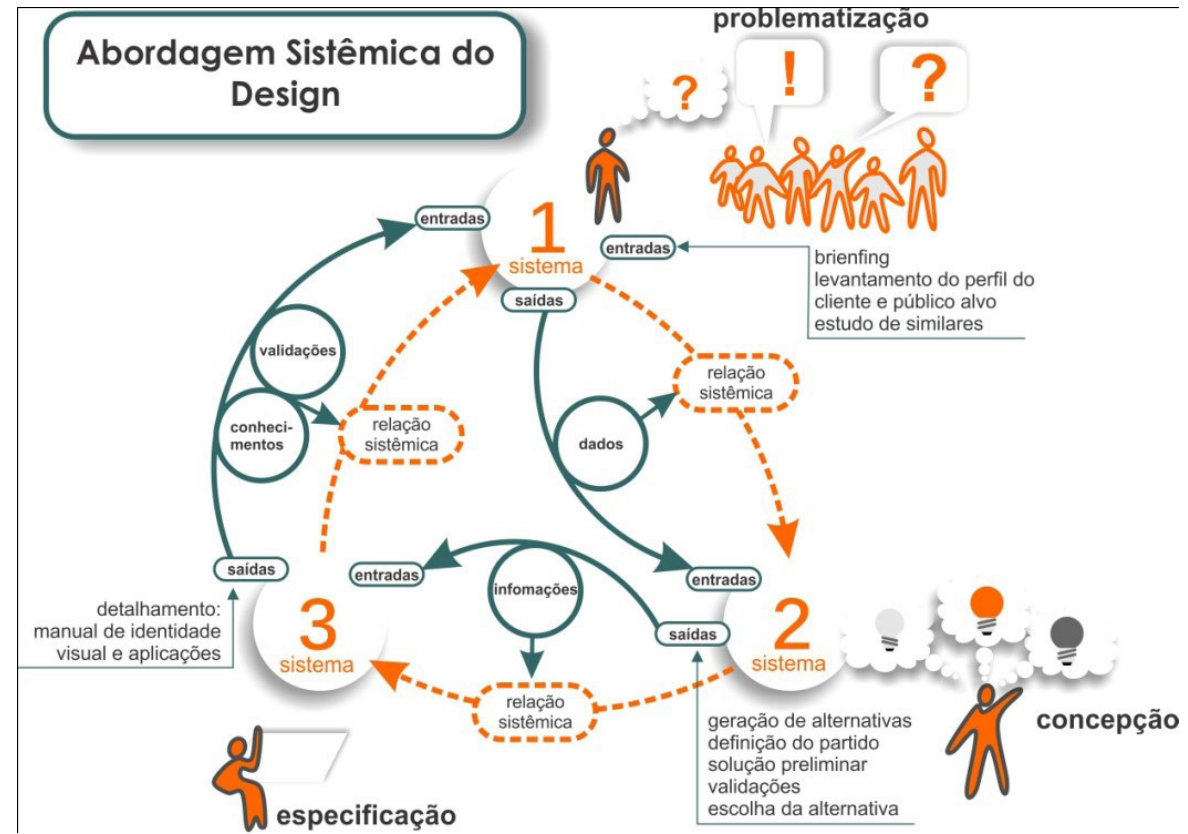

Como mostra a Figura 3, a abordagem sistêmica pode ser aplicada em metodologias de projeto de design, sejam gráficos, de produto, etc. Em vista disso, percebe-se que o objetivo do NASDesign é propor soluções sistêmicas, por meio de um processo de ensino-aprendizagem, que satisfaçam as necessidades das comunidades, favoreçam a prática de iniciativas locais e permitam que atores sociais desenvolvam uma consciência sustentável. "Estratégias de relacionamento em rede tem a vantagem dual de ser capaz de produzir experiências positivas e significativas ao mesmo tempo para a comunidade e para o indivíduo." (MERONI, 2007, p. 10).

A abordagem sistêmica está apoiada no postulado da interdependência no qual se destaca o relacional antes dos objetos. Para melhor compreender um sistema, pode-se dizer que ele é constituído de elementos estruturados em subsistemas, entre os quais as relações de trocas contínuas (entradas e saídas) geram fluxos de: 
energia, matérias, informações, decisões etc.

Dessa forma, justifica-se a sistematização das atividades com intuito de entender como o Núcleo pode buscar a implementação bem sucedida de suas ações, alinhando-as e integrando-as aos seus objetivos e metas estratégicas focadas no ensino, na pesquisa e extensão. Sendo assim, a sistematização representa uma articulação entre teoria e prática, e serve aos objetivos dos dois campos.

Nessa perspectiva, a construção de uma sistematização tem como objetivo levantar áreas de interesse, ampliando então, a capacidade de identificar demandas tangíveis e intangíveis na contextualização dos projetos.

Então, torna-se essencial enfatizar que estas etapas não ocorrem de forma linear e isolada, mas de modo entrelaçado, no qual também é permitido retornar as fases já transpostas ou administrá-las simultaneamente.

Figura 4 - sistematização das atividades do NASDesign.

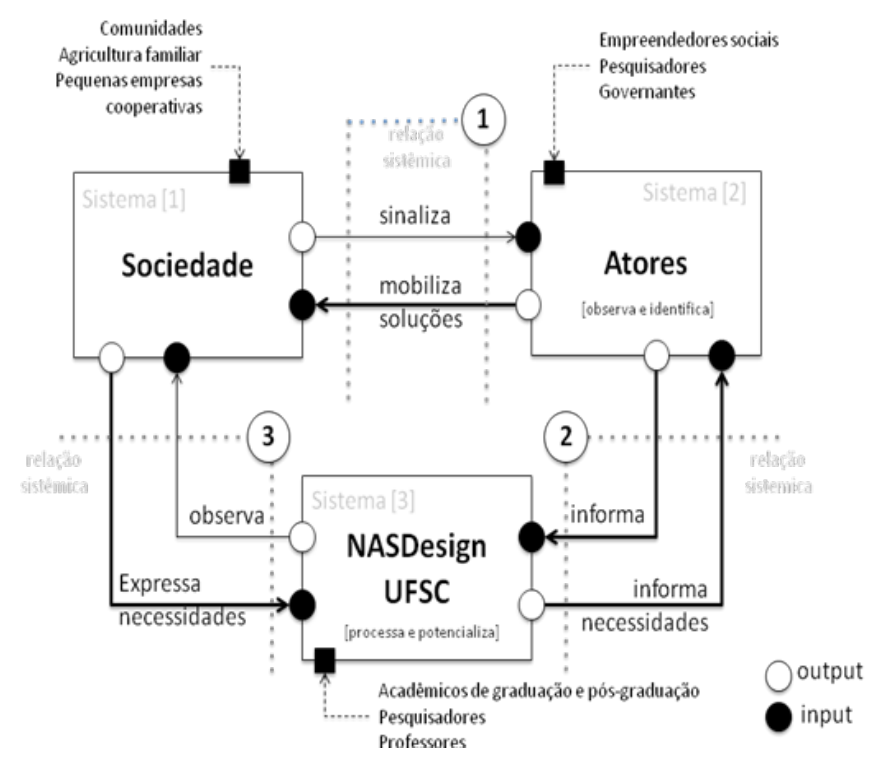

\section{CONSIDERAÇÕES FINAIS}

O objetivo do NASDesign consisti em fortalecer a identidade desses grupos produtivos, associações e cooperativas de Santa Catarina, por meio do Processo de Design Habilitante para Iniciativas Locais, a fim de melhorar o posicionamento de seus produtos no mercado e valorizar as raízes de suas tradições locais. Com este processo, foi possível construir vivências junto às comunidades, no intuito de interpretar características subjetivas que representavam as experiências dessas famílias, em seus valores e estética. $O$ resultado foi a conformação de identidade visual para todas, como um brasão da produção familiar, o que trouxe motivação social e sentimento de pertencimento, além da maior aceitação às práticas de design. Com esta abertura, fica muito mais fácil inserir outros tipos de programas sociais, como a inserção de novos serviços, interfaces e ferramentas que melhor organizam as produções a uma realidade sustentável.

Esses projetos foram elaborados com o intuito de dar unidade ao conjunto de famílias que se identificam com suas marcas próprias, mas que também procuram 
fazer parte de um conjunto maior. Isto facilita o entendimento da formação do grupo com a mesma identidade, o que proporciona o sentimento de pertencimento às famílias e facilita a relação entre as pessoas em troca de serviços futuros.

\section{CONSTATAÇÕES / RESULTADOS}

Além da rede, os resultados da sistematização das atividades de projeto em grupos produtivos são marcas locais, Sistemas de identidade visual, embalagens, catálogos e brochuras de produtos, blogs, páginas de fãs, sinalizando o local de trabalho, uniformes, equipamentos de desenvolvimento, entre outros.

\section{REFERÊNCIAS}

Baxter, M. Product Design: A practical guide for the development of new products. Sao Paulo: Edgar

Blücher, 1998.

Bürdek, B. E. Design: history, theory and practice of designing products. Sao Paulo: Edgard Blucher, 2006.

Coelho, L. A. L. (ed.). Key concepts in design. Rio de Janeiro: Ed PUC-Rio New Ideas, 2008.

Gil, A. C. How to design research projects. Sao Paulo: Atlas, 2007.

Kasper, H. Systems thinking. [S.L.: S.N.], 2006.

Vassão, C. A. Metadesign - Thinking the Design Collection. Publisher Blucher, 2010. 